\title{
Disordered Ground States for Classical Discrete-state Problems in One Dimension
}

G Canright and G Watson

May 1995 
(ㄷ) Council for the Central Laboratory of the Research Councils 1995

Enquiries about copyright, reproduction and requests for

additional copies of this report should be addressed to:

The Central Laboratory for the Research Councils

Library and Information Services

Rutherford Appleton Laboratory

Chilton

Didcot

Oxfordshire

OX11 OQX

Tel: 01235445384 Fax: 01235446403

E-mail library@rl.ac.uk

ISSN 1358-6254

Neither the Council nor the Laboratory accept any responsibility for loss or damage arising from the use of information contained in any of their reports or in any communication about their tests or investigations. 


\title{
Disordered Ground States for Classical Discrete-state Problems in One Dimension
}

\author{
Geoff Canright ${ }^{1,2}$ and Greg Watson ${ }^{3}$ \\ ${ }^{1}$ Department of Physics and Astronomy \\ The University of Tennessee, Knoxville, TN 37996 \\ 2 Solid State Division, Oak Ridge National Laboratory \\ Oak Ridge, Tennessee 37831 \\ ${ }^{3}$ Rutherford Appleton Laboratory \\ Chilton, Didcot, Oxon OX11 0QX, U.K.
}

\begin{abstract}
It is known that one-dimensional lattice problems with a discrete, finite set of states per site 'generically' have periodic ground states (GSs). We consider slightly less generic cases, in which the Hamiltonian is constrained by either spin $(S)$ or spatial $(I)$ inversion symmetry (or both). We show that such constraints give rise to the possibility of disordered GSs over a finite fraction of the coupling-parameter space-that is, without invoking any nongeneric 'fine tuning' of coupling constants, beyond that arising from symmetry. We find that such disordered GSs can arise for many values of the number of states $(k)$ at each site, and the range $r$ of the interaction. The Ising $(k=2)$ case is the least prone to disorder: $I$ symmetry allows for disordered GSs (without fine tuning) only for $r \geq 5$, while $S$ symmetry 'never' gives rise to disordered GSs.
\end{abstract}




\section{INTRODUCTION}

The problem of order vs. disorder permeates all of condensed-matter and statistical physics. If we ignore thermal fluctuations by setting $T=0$, and quantum fluctuations as well by viewing matter as composed of massive units interacting via effective classical potentials, we have a simpler problem which is still nontrivial. Here we want to consider the simplest subproblem of this class: we restrict our units to lie on a one-dimensional chain, and allow them only a finite, discrete set of states, whose number we call $k$. We take the (integer) range of the interaction among the units to be $r$, but do not restrict the interactions to two-body terms. (We will call the units 'spins'.) The Hamiltonian is then of the form

$$
H=\sum_{i} f\left(\sigma_{i}, \sigma_{i+1}, \ldots, \sigma_{i+r}\right)
$$

where $\sigma_{i}$ (the spin at site $i$ ) has $k$ states which we label $0,1, \ldots,(k-1)$, and we assume an infinite chain.

The question of ordered/disordered ground states (GSs) for this problem has already been answered in principle. Radin and Schulman (RS) ${ }^{1}$ showed that (i) a nondegenerate GS is periodic, and (ii) in the case of degenerate GSs, there always exists at least one periodic GS. (See also Teubner ${ }^{2}$ for a different presentation of the same results for the Ising case $k=2$.) In each case the maximum period of the periodic GS is $k^{r}$.

These results may be understood most simply by embodying the information contained in the Hamiltonian $H$ in a directed graph $G_{r}^{(k)}$ (where $k$ and $r$ have the same meanings as above). This is done as follows: $:^{2,3}$ nodes of the graph are sets of $r$ spins, each taking one of the $k$ values. A directed arc points from node $\mathcal{N}_{1}$ to node $\mathcal{N}_{2}$ whenever the rightmost $(r-1)$ values of $\mathcal{N}_{1}$ agree with the leftmost $(r-1)$ values of $\mathcal{N}_{2}$. The arc itself may then be uniquely labeled with $(r+1)$ sequential spin values, which allow us to associate a unique weight (energy) to the arc. Specifically, we can take the weight of the arc joining the node $\left(\sigma_{i}, \sigma_{i+1}, \ldots, \sigma_{i+r-1}\right)$ to the node $\left(\sigma_{i+1}, \sigma_{i+2}, \ldots, \sigma_{i+r}\right)$ to be $f\left(\sigma_{i}, \sigma_{i+1}, \ldots, \sigma_{i+r}\right)$. The graph $G_{r}^{(k)}$ has $k^{r}$ nodes, and $k^{r+1}$ arcs. Any configuration of an infinite chain of spins thus must involve repeated cycles of $\operatorname{arcs}$ in $G_{r}^{(k)}$. 
All cycles in $G_{\leftarrow}^{(k)}$ may be decomposed into 'simple cycles' $(\mathrm{SCs})^{4}$ having the property of non-self-intersection. The result of Radin and Schulman is then equivalent to the following: (i) If there is a unique SC of $G_{r}^{(k)}$ with the lowest weight per spin, then a repetition of that SC gives the nondegenerate GS, whose period (the length of the SC) is $\leq$ the number of nodes of the graph, ie, $k^{r}$. (ii) If there are two or more SCs with the lowest weight per spin, then there is always a GS consisting of repetitions of only one of them, whose period is again $\leq k^{r}$

This logic does not allow a reduction of the upper bound of RS. The graphs $G_{\tau}^{(k)}$ are known as de Bruijn graphs; ${ }^{5,6}$ and it is known that the set of SCs always includes a 'Hamiltonian cycle', that is, one visiting all the nodes. Furthermore, it is clear that case (ii) is 'rare': in general - that is, without fine tuning of the couplings to precise values - the Hamiltonian $H$ does not give degenerate SCs.

We also note that, in the exceptional case of degenerate (and minimal-weight) SCs, the degeneracy may give rise to disordered GSs if the degenerate SCs share one or more nodessince the different SCs may then be traversed in any arbitrary sequence with no energy cost.

Put briefly, we see that, barring fine tuning of the coupling parameters of $H$, one always has a periodic GS for a $k$-state model. Given such fine tuning, however, one can arrive at a degenerate set of GSs, with this set (sometimes, apparently) infinitely large, and including disordered configurations.

Given this background, we note the following: sometimes, 'fine tuning' is 'generic'. By this we mean simply that symmetry in fact does tune some parameters to precise values. This suggests the following question: might the symmetries of $H$ give rise to disordered, degenerate GSs - without any further fine tuning of parameters?

We offer an answer to this question here. The symmetries we consider are two: spin inversion $(S)$ symmetry, and spatial inversion $(I)$ symmetry. We choose these two because they are both (particularly the latter) very common in physical applications. (We should 
also point out that our entire analysis, like that of $\mathrm{RS}$, assumes translational invariance of the Hamiltonian as well; put differently, we are seeking disordered GSs in the absence of quenched disorder in the Hamiltonian.)

Our approach is as follows. We will construct, for a given symmetry $X(=S, I$, or combinations of the two), a symmetry-reduced graph ${ }^{x} G$ which will play the same role in the case of a symmetry-constrained Hamiltonian $H^{X}$ as is played by the full graph $G$ for the generic Hamiltonian $H$. That is, we define a graph ${ }^{x} G$, and its SCs, such that the set of SCs of ${ }^{X} G$ is the set of GSs of $H^{X}$. This will allow us to enumerate explicitly those $(k, r)$ values for which there are disordered GSs in the set. The results (for $X=S$ and $I$ ) are shown in Table I, which is the principal result of this paper. A second result is that our construction of ${ }^{x} G$ enables an explicit, algorithmic enumeration of all the GSs of a given $H^{x}$.

Finally, a technical point, which is implicit in the preceding discussion. Following Teubner, ${ }^{2}$ we define a GS to be a spin configuration which is the lowest-energy configuration over a finite subvolume of coupling-parameter space. That is, we only include configurations which do not require fine tuning of the parameters (beyond that coming from the assumed symmetry). Such configurations may also be termed zero-temperature phases, since they occupy a finite region in parameter space.

\section{BROKEN SYMMETRY AND DISORDER}

Broken symmetry is by now a well-known idea, with a canonical example from our own problem class: the near-neighbor Ising problem $(k=2, r=1)$ in zero field is $S$-symmetric, but may have two degenerate ferromagnetic GSs, each breaking the symmetry. There is however in this example no obvious connection to disorder: one does not find a disordered mixture of the two phases in the GS manifold, because the two GSs have a finite 'surface tension' between them-which translates to the fact ${ }^{2}$ that the two SCs do not share nodes in $G_{1}^{(2)}$.

To find disordered GSs, then, we seek symmetry-degenerate SCs of ${ }^{x} G$ which do share 
nodes - that is, degenerate GSs with zero surface tension. One can of course readily find pairs of SCs in $G_{r}^{(k)}$ which are symmetry-related (hence degenerate under a symmetric Hamiltonian) and which share one or more nodes. The problem is then to determine which (if any) of these pairs are ground states, by our definition. What we find is that the imposition of symmetry, while nicely enforcing the degeneracy of pairs of cycles over the entire (symmetric) coupling-parameter space, can also-due to the degeneracy of parts of the pairs-suggest the 'decomposition' of the pair into two or more other cycles, which are not related by symmetry, and one of which must be lower in energy than the degenerate pair. Decomposition in this sense of a degenerate pair of cycles, when it occurs, excludes that pair from our set of GSs of $H^{X}$.

Hence the problem is to find symmetry-related pairs of cycles which share one or more nodes but do not decompose. (We will give explicit examples of decomposition below.) Our construction of ${ }^{x} G_{r}^{(k)}$, and our definition of its SCs, are designed to solve this problem. Below we show in detail how this is accomplished, for various combinations of $\operatorname{spin}(S)$ and space $(I)$ inversion symmetry.

\section{A. Spin inversion $(S)$}

By spin inversion for $k$-state problems we mean the following: the states, which we formally label $(0,1, \ldots, k-1)$, map under $S$ to $(k-1, k-2, \ldots, 0)$. Nodes, arcs, and cycles of the graph $G_{r}^{(k)}$ also map to their spin-inverses: $\mathcal{N} \rightarrow \overline{\mathcal{N}}$, arc $\rightarrow \overline{\operatorname{arc}}$, and $c y c \rightarrow \overline{c y c}$. We recall that the energetics of our discrete problem is reflected in the weights $w$ assigned to the arcs of the graph $G_{r}^{(k)}$; the symmetry of $H$ is then reflected in $w(\operatorname{arc})=w(\overline{a r c})$ and hence $w(c y c)=w(\overline{c y c})$.

The SCs of $G$ are of course unchanged by the application of symmetry; but the set of GSs is reduced in number. The GSs of the general problem $G_{r}^{(k)}$ are vertices of a convex polytope $P_{\tau}^{(k)}$ in a $d$-dimensional space (where $d$ is the number of independent spin correlations, and hence coupling parameters, in $H$ ); these vertices are in one-to-one correspondence ${ }^{2}$ with 
the SCs of $G$. Applying $S$ symmetry to $H$ then amounts to constraining $H$ to move in a lower- $\left(d^{S}\right)$-dimensional subspace. The GSs of the symmetric Hamiltonian $H^{S}$ are then the vertices of the section ${ }^{7}$ of $P_{r}^{(k)}$ defined by the lower-dimensional space; we call this section ${ }^{s} P_{\tau}^{(k)}$. The vertices of ${ }^{s} P_{r}^{(k)}$ correspond to either a symmetric SC, or an $S$-related pair of SCs. In the latter case, if the pair shares one or more nodes, it will give rise to an infinite set of degenerate GSs, 'most' of which are disordered mixtures of the two SCs.

Hence our original question may now be rephrased as: for which $k$ and $r$ values is there at least one vertex of ${ }^{s} P_{r}^{(k)}$ corresponding to a pair of SCs sharing at least one node? To answer this question, we turn to the graph ${ }^{s} G_{\tau}^{(k)}$, which is more readily visualized.

We would like to find a graph ${ }^{S} G_{r}^{(k)}$ with the same properties, with respect to $H^{S}$, that $G_{r}^{(k)}$ possesses with respect to $H$ : all GSs are SCs, and all SCs are GSs. We consider the following construction ${ }^{8}$ (Fig. 1). We identify the equal-weight $\operatorname{arcs} \operatorname{arc}$ and $\overline{\operatorname{arc}}$ of $G_{r}^{(k)}$ with the single arc arc $c^{s}$ of ${ }^{s} G_{r}^{(k)}$; similarly, we merge the nodes $\mathcal{N}$ and $\overline{\mathcal{N}}$ to a single node $\mathcal{N}^{s}$. The resulting graph, for $k=2$, has the nice property (as may be guessed from Teubner ${ }^{2}$ ) that ${ }^{s} G_{r}^{(2)} \sim$ (is isomorphic to) $G_{r-1}^{(2)}$. For larger $k,{ }^{s} G_{r}^{(k)}$ is in general no longer a de Bruijn graph, since it includes parallel arcs.

We define a SC of ${ }^{S} G_{r}^{(k)}$ in precise analogy to a SC of $G_{r}^{(k)}$; it is a cycle which visits no node in ${ }^{S} G_{r}^{(k)}$ more than once.

Now we want to show that only SCs of ${ }^{s} G_{F}^{(k)}$ can be GSs of $H^{S}$. Clearly it is sufficient to restrict our attention to non-SCs of ${ }^{s} G_{r}^{(k)}$ which are SCs of $G_{r}^{(k)}$, since we have already ruled out non-SCs of $G_{r}^{(k)}$. A SC of $G_{r}^{(k)}$ (visiting no node in $G_{r}^{(k)}$ twice), which is however a non-SC of ${ }^{s} G_{r}^{(k)}$, will visit at least one node $\mathcal{N}^{s}$ in ${ }^{s} G_{r}^{(k)}$ exactly twice [Fig. 2(a)]. In $G_{r}^{(k)}$, this non-simple cycle of ${ }^{s} G_{r}^{(k)}$ represents a SC cyc, and its partner $\overline{c y c}$. Schematically [Fig. 2(b)] we can represent these cycles as

$$
\begin{array}{ll}
\text { cyc }: & \mathcal{N} \stackrel{b / m}{\longrightarrow} \overline{\mathcal{N}} \stackrel{a / \ell}{\longrightarrow} \mathcal{N} \\
\overline{c y c}: & \bar{N} \stackrel{b / m}{\longrightarrow} \mathcal{N} \stackrel{a / \ell}{\longrightarrow} \mathcal{N} .
\end{array}
$$

Here the long arrows each represent a path (a composition of arcs), and the energy per 

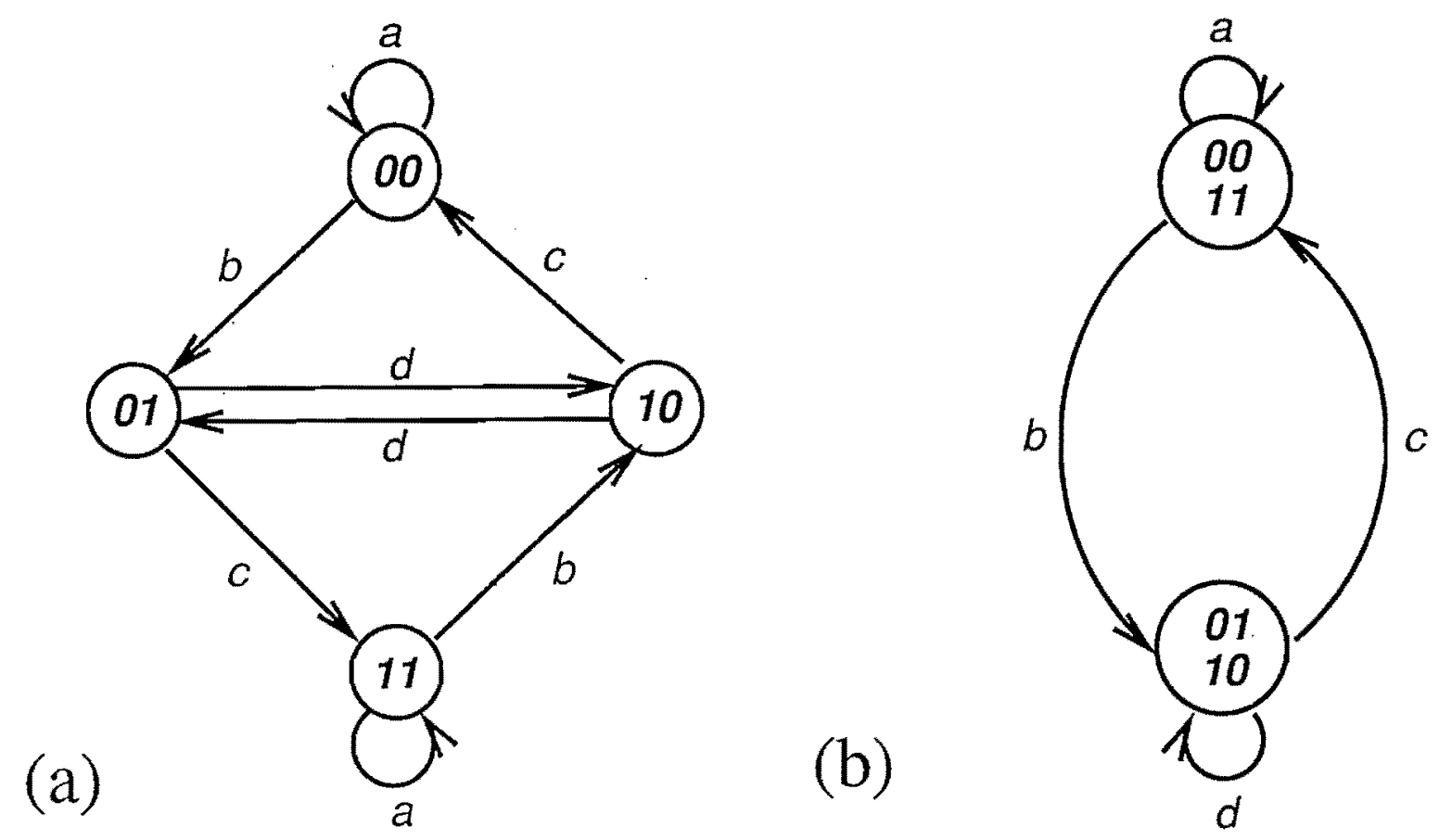

FIG. 1. The graphs (a) $G_{2}^{(2)}$ and (b) ${ }^{s} G_{2}^{(2)}$. Arcs which have equal weight by $S$ symmetry are given the same label. Note that the latter graph is isomorphic to $G_{1}^{(2)}$ (Fig. 6).

spin of each path is placed above the arrow. The energy per spin of $c y c$ and $\overline{c y c}$ is then $(a+b) /(\ell+m)$. It is apparent from Fig. 2(b) that cyc and $\overline{c y c}$ together define two other cycles: one with energy $/ \operatorname{spin} a / \ell$, the other with $b / m$. We assume that $a / \ell<b / m$. We then use the fact that

$$
a / \ell<(a+b) /(\ell+m)<b / m
$$

to deduce that the non-SC $c y c / \overline{c y c}$ of ${ }^{S} G_{r}^{(k)}$ is not a GS of $H^{S}$ : barring fine tuning of parameters (as would be needed to set $a / \ell=b / m$ ), one of the symmetric cycles is always lower in energy. Alternatively, we say that the non-SC cyc/ $\overline{c y c}$ decomposes to the two cycles with intensive energy $a / \ell$ and $b / m$. This conclusion holds without any restriction on the number of nodes which may be shared between the $a / \ell$ and $b / m$ paths-which therefore also may decompose.

Hence we find that all cycles of ${ }^{S} G_{r}^{(k)}$ which are not SCs of ${ }^{s} G_{r}^{(k)}$ are not GSs of $H^{S}$. Now we wish to show that all the SCs of ${ }^{s} G_{r}^{(k)}$ are GSs of $H^{S}$. It is helpful to recall the generic case first, since the argument is then readily generalized to the case of $S$ symmetry. 


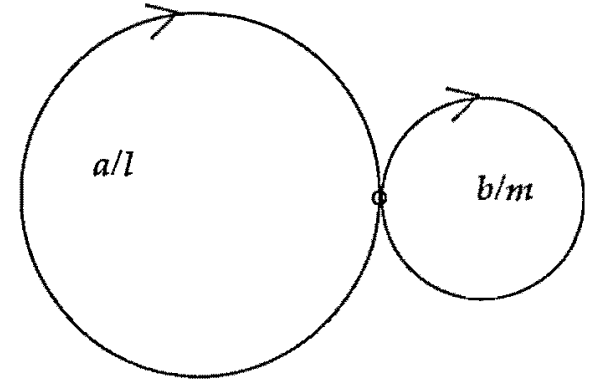

(a)

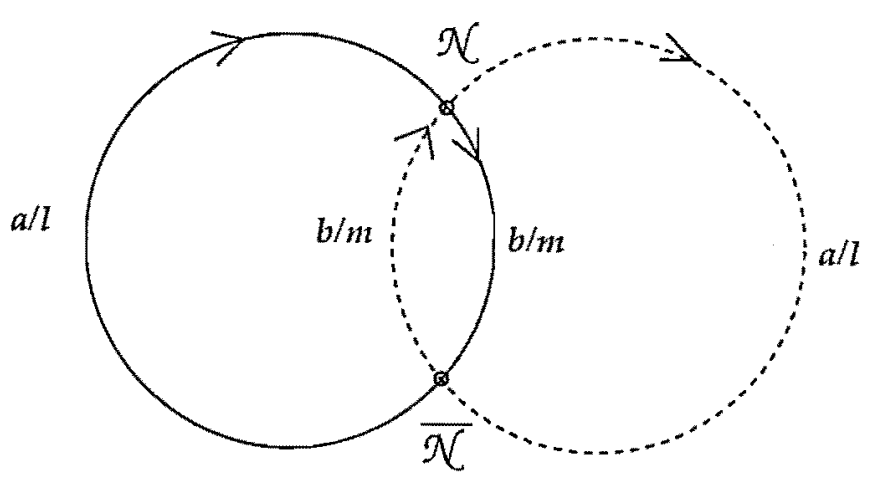

(b)

FIG. 2. (a) A (schematic) non-simple cycle of ${ }^{s} G_{r}^{(k)}$, composed of two simple cycles (SCs) whose per-spin weights are marked. (b) How the non-SC appears in $G_{r}^{(k)}$ : as two, asymmetric but symmetry-related, SCs (one solid, one dashed), each of net weight/spin $(a+b) /(\ell+m)$. The single shared node in (a) becomes two symmetry-related nodes $\mathcal{N}$ and $\mathcal{N}$ in (b). The asymmetric SCs are never ground states of an $S$-symmetric Hamiltonian (see text).

We consider the graph $G_{r}^{(k)}$, and the corresponding polytope $P_{r}^{(k)}$. The vertices of $P_{r}^{(k)}$ represent extrema of the Hamiltonian and hence GSs. Since the polytope $P_{r}^{(k)}$ is defined ${ }^{2}$ by the intersection of a set of inequalities (of number $n_{f}$ ) on the correlations of $H$, the faces of $P_{r}^{(k)}$ are a set of $n_{f}$ equalities. As shown by Teubner, ${ }^{2}$ the inequalities for the correlations may be derived from the simpler inequalities for the densities $n_{\sigma}$. The density $n_{\sigma}$ is simply the average occurrence of the $\operatorname{arc} \sigma \equiv\left\{\sigma_{1} \sigma_{2} \ldots \sigma_{\tau+1}\right\}$ in a given infinite configuration of spins $\sigma_{i}$. The inequality is then

$$
n_{\sigma} \geq 0
$$

Hence $n_{f}$ is simply the number of distinct densities, where 'distinct' means 'not constrained to be equal'.

Constraints on the densities arise in the following way. Since the 'flow' in the graph $G_{r}^{(k)}$ is 'incompressible', densities for the case where a single arc enters a node, and a single arc leaves it, are forced to be equal. This flow-induced constraint must be accounted for in order to count correctly the faces of the correlation polyhedron. ${ }^{2}$ 
Now consider a SC of $G_{\tau}^{(k)}$. Since it cannot visit every arc, it satisfies a set of equalities of the form $n_{\sigma_{i}}=0, i=1 ; \ldots, n_{e}(\mathrm{SC})$ (where $n_{e}(\mathrm{SC})$ is the number of distinct, unvisited arcs). Hence the SC is represented in $P_{\tau}^{(k)}$ by the locus of points satisfying that set of equalities. This locus lies on the surface of $P_{r}^{(k)}$ because $n_{e}(\mathrm{SC}) \geq 1$. Furthermore, the locus is a point, since all densities in a SC are fixed (to $1 / p$, where $p$ is the period of the $\mathrm{SC}$ ); and, since varying any nonzero density of a $\mathrm{SC}$ requires the inclusion of arcs whose density was zero, every neighborhood of the point represented by a SC violates one or more of the equalities satisfied at the point. Therefore, the SC corresponds to a vertex of $P_{r}^{(k)}$, hence to a GS of $H$.

Now consider the restriction to $H=H^{S}$, with corresponding polytope ${ }^{s} P_{\tau}^{(k)}$. A further constraint on the densities arises when we project $P_{r}^{(k)}$ to the lower-dimensional, symmetryinvariant subspace. In this subspace, a configuration and its symmetry-related partner give rise to the same point, since they have the same symmetric correlations. Put more simply, a correlation $\left\langle\sigma_{1}^{p_{1}} \sigma_{2}^{p_{2}} \ldots \sigma_{q}^{p_{q}}\right\rangle$ and its symmetry partner (obtained from $\sigma_{i} \rightarrow \overline{\sigma_{i}}$ ) represent the same coordinate in the projected subspace. Since the densities represent a linear transformation on the correlations, it follows that the densities $n_{\sigma}$ and $n_{\bar{\sigma}}$ are also identified in the invariant subspace. Hence $n_{\sigma}$ and $n_{\bar{\sigma}}$ cannot be considered distinct when we work in the invariant subspace.

Now consider a SC of ${ }^{S} G_{r}^{(k)}$. The argument is essentially the same as for the generic case. The (locus of the) SC lies on the surface of ${ }^{s} P_{r}^{(k)}$, since the SC cannot visit all the $\operatorname{arcs}$ of ${ }^{S} G_{\tau}^{(k)}$. And the distinct (with the above constraint, $n_{\sigma}$ not distinct from $n_{\bar{\sigma}}$ ) densities of the SC are fixed by the fact that the SC represents an unambiguous path in ${ }^{s} G_{r}^{(k)}$. Finally, varying the densities from those of a SC again requires violating one or more further equalities. Hence, SCs of ${ }^{s} G_{r}^{(k)}$ are vertices of ${ }^{s} P_{r}^{(k)}$, and so are GSs of $H^{s}$.

Hence we have shown that the SCs of ${ }^{s} G_{\tau}^{(k)}$ are the GSs of $H^{s}$. Our search for disordered GSs now takes the form: when does a SC of ${ }^{s} G_{r}^{(k)}$ represent a pair of node-sharing SCs of $G_{r}^{(k)}$ ? 
The question in this form can be answered, and the answer is simple. We call a 'nodesharing' pair in $G_{r}^{(k)}$ (which is represented by a single SC of ${ }^{s} G_{r}^{(k)}$ ) a 'D-pair', where D stands for 'degenerate and disordered'. Then a SC of ${ }^{s} G_{r}^{(k)}$ represents a D-pair only if it includes an $S$-invariant node $\mathcal{N}^{*}$. Such a cycle (excluding the 'ferromagnetic' SC which uses the arc $\left.\mathcal{N}^{*} \rightarrow \mathcal{N}^{*}\right)$ maps to a pair of cycles in $G_{\boldsymbol{r}}^{(k)}$, sharing the node $\mathcal{N}^{*}$. All other SCs of ${ }^{s} G_{\boldsymbol{r}}^{(k)}$ map to either a single, symmetric cycle (of twice the period) in $G_{r}^{(k)}$, or to a pair of SCs sharing no nodes. (As shown above, no $\mathrm{SC}$ of ${ }^{s} G_{r}^{(k)}$ maps to a pair sharing two or more nodes.)

The graph $G_{\tau}^{(k)}$ contains no $S$-invariant nodes for $k$ even, and one such node [consisting of $r$ consecutive occurrences of the invariant spin value $\left.\sigma^{*}=(k-1) / 2\right]$ for odd $k$. Furthermore, given odd $k$, there is always (ie, for any $r$ ) at least one SC of ${ }^{S} G_{r}^{(k)}$ which uses the node $\mathcal{N}^{*}$. Hence we conclude the following:

For even $k, S$ symmetry never gives rise to disordered GSs; for odd $k$ and for every $r, S$ symmetry does give rise to disordered GSs.

\section{B. Space inversion $(I)$}

\section{Preliminaries}

The arguments and conclusions with respect to $I$ symmetry are somewhat more involved than those for spin inversion. However, the basic outline of the argument is the same: we wish to define a graph ${ }^{I} G_{r}^{(k)}$ whose SCs are the GSs of $H^{I}$ (that is, the Hamiltonian $H$ constrained to be invariant under $I$ ). We will then seek D-pairs among the SCs of ${ }^{I} G_{r}^{(k)}$. The definition of ${ }^{I} G_{r}^{(k)}$, and of its SCs, will be developed in this subsection, along with a number of auxiliary concepts which are useful for the argument. We follow this subsection with a search for D-pairs, arising from $I$ symmetry, in $k$-state problems with $k>2$. Finally, in the last subsection, we treat the special (Ising) case $k=2$. 
Classification schemes and representations:- We begin by developing a classification scheme for the nodes and arcs of $G_{\boldsymbol{r}}^{(k)}$ according to their behavior under $I$. All the nodes of $G_{r}^{(k)}$ may be classified, according to their behavior under $I$, into three sets: a 'left-handed' set given the label $L$, their space inverses $R$, and an invariant or symmetric set labelled with $S$. Since the arcs of $G_{r}^{(k)}$ are in one-to-one correspondence with the nodes of $G_{r+1}^{(k)}$, the same holds true for the arcs.

Clearly there are, in general, many ways of choosing the $L$ and $R$ sets. Each such choice can be taken as a constraint on how the graph $G_{r}^{(k)}$ is to be represented in a planar drawing (eg, $R$ nodes on the right, $L$ nodes on the left). We are of course most interested in those properties of $G_{r}^{(k)}$, and of ${ }^{I} G_{r}^{(k)}$, which are independent of the choice of representation. However we will find two types of representation (or rep, for brevity) to be most convenient.

First we define the 'recursive' representations. (For $k=2$ there are two; for general $k$ there are many.) In these representations the handedness of all nodes and arcs of $G_{r}^{(k)}$ are determined, as much as possible, from the handedness of the arcs of $G_{1}^{(k)}$. Hence, one must first choose a classification for the arcs of $G_{1}^{(k)}$. (The nodes, of length $r=1$, are all inversion-invariant and so $S$.)

One then exploits a representation-independent procedure ${ }^{6}$ for constructing $G_{r+1}^{(k)}$ from $G_{r}^{(k)}$. [Such a procedure gives (by recursion) $G_{r}^{(k)}$, for any $r$, from $G_{1}^{(k)}$ ]. The arcs of $G_{r}^{(k)}$, representing all the possible $k^{r+1}$ sets of $r+1$ spins, become the nodes of $G_{r+1}^{(k)}$. Arcs of $G_{r+1}^{(k)}$ then represent adjacent sets of $r+2$ spins, which may be traced back to adjacent pairs of arcs in $G_{r}^{(k)}$ (where 'adjacent' means meeting at a node, with one arc of the pair incoming, the other outgoing).

Our 'recursive' reps then use the following rules. Since nodes of $G_{r+1}^{(k)}$ come from arcs of $G_{r}^{(k)}$, we carry the handedness through unchanged. The handedness of the arcs of $G_{r+1}^{(k)}$ is then determined from that of the corresponding arc pairs of $G_{r}^{(k)}$ as follows:

$$
\begin{array}{r}
R R \rightarrow R \\
S R \text { or } R S \rightarrow R
\end{array}
$$




$$
\begin{aligned}
& L L \rightarrow L \\
& S L \text { or } L S \rightarrow L \\
& S S \rightarrow S, L, \text { or } R \text { (= inverse of intervening node) } \\
&R L \text { or } L R \rightarrow S \text { (if } R \text { and } L \text { are related by } I) \\
& R L \text { or } L R \rightarrow J \text { otherwise }
\end{aligned}
$$

The last line needs of course clarification. We use the symbol $J$ to mean a 'joining arc' (JA): an arc that joins an $R$ node to an $L$ node, but is not symmetric itself. We note that symmetry, or lack of same, is rep-independent; however whether or not a given arc is $J$ is rep-dependent, since one can always move one of the nodes. Joining arcs will be significant in many parts of our discussion of $I$ symmetry. Specifically, the proper treatment of JAs is an essential part of our definition of ${ }^{I} G_{r}^{(k)}$ and its SCs; also, the concept of JAs is the simplest way to understand our results for $k=2$, for which we will distinguish the cases $r<5$ from those with $r \geq 5$. Note that, for the purpose of proceeding with a recursive rep, the handedness of a JA must be chosen arbitrarily to be $L$ or $R$.

This completes our description of recursive reps. We now define a second type of representation, a 'minimal' rep, as follows. In a minimal rep the number $n_{J}$ of JAs is minimized. That is, all minimal reps have the same $n_{J}$, and every rep that is not minimal has a larger $n_{J}$.

We can always draw the graph $G_{r}^{(k)}$ in the plane such that it is reflection symmetric about a line $\mathcal{I}$ (the reflection accomplishing $L \leftrightarrow R$ for both nodes and arcs). If there are no JAs, the line $\mathcal{I}$ then partitions $G_{\tau}^{(k)}$, drawn in this way, into disjoint sets of arcs and nodes, with $R$ and $L$ arcs/nodes on opposite sides of $\mathcal{I}$, and only $S$ arcs/nodes touching $\mathcal{I}$. Thus if $n_{J}=0$ (in any rep), we call the graph " $\mathcal{I}$-disjoint". If $n_{J} \neq 0$ in a minimal rep, the graph is non- $\mathcal{I}$-disjoint. Obviously, $\mathcal{I}$-disjointness is a property which is independent of rep, but most easily ascertained in a minimal rep.

Not all minimal reps are recursive. For example, for the case $k=2$, and in the absence 
of JAs, there are only two recursive reps. These two reps are trivially related, since there are only two arcs in $G_{1}^{(2)}$ (cf Fig. 8) which are not $S$-and they must have opposite handedness. Since the relation of the two is trivial, then, as long as JAs do not appear to introduce ambiguity in going from $(r-1)$ to $r$, we can refer to a 'single' recursive rep for $k=2$. We find, by construction, for $k=2$ that 'the' recursive rep is also minimal for all $r \leq 5$. [This is the reason for the rule for $S S$ combinations, Eq. 6(e).] However, for $r=6$ there are multiple recursive reps due to the appearance of JAs at $r=5$; and no recursive rep is minimal. These results, besides demonstrating that minimal reps are not in general recursive, will be useful in our discussion of the $k=2$ case below.

The graph ${ }^{I} G$ and its $S C$ s:- We now seek the graph ${ }^{I} G_{r}^{(k)}$ which will serve the same purpose, in the case of $I$ symmetry, as was served by ${ }^{s} G_{\tau}^{(k)}$ for $S$ symmetry. Unfortunately, it is impossible ${ }^{8}$ to draw consistently a graph strictly analogous to ${ }^{s} G_{r}^{(k)}$, that is, a graph in which arcs and nodes related by $I$ are identified.

We can however construct ${ }^{I} G_{r}^{(k)}$ by broadening our notion of a graph (and of a cycle). We draw $G_{r}^{(k)}$ to be reflection-symmetric about the symmetry line $\mathcal{I}$ as described above, with $L$ arcs and nodes to the left, $R$ to the right, $S$ nodes on $\mathcal{I}$, and $S$ (and $J$ ) arcs crossing $\mathcal{I}$. We then construct ${ }^{I} G_{r}^{(k)}$ by simply erasing everything to one side of $\mathcal{I}$ (Fig. 3). The resulting 'graph' (we will drop the quotes) has the odd property that some arcs begin and end on $\mathcal{I}$, rather than on a node. We define a 'cycle' of ${ }^{I} G_{r}^{(k)}$ to be one of two types: (i) a closed path as in a conventional digraph, or (ii) a path which begins and ends on $\mathcal{I}$. A cycle of type (i) will map to two distinct ( $I$-related) cycles in $G_{r}^{(k)}$. A type-(ii) cycle becomes a cycle in $G_{r}^{(k)}$ by simple reflection about $\mathcal{I}$.

Our prescription for ${ }^{I} G_{\tau}^{(k)}$ is still not complete; joining arcs in $G_{\tau}^{(k)}$ require special handling. Assume the JA arc connects nodes $\mathcal{N}_{L} \rightarrow \mathcal{N}_{R}$ in $G_{r}^{(k)}$, that $\mathcal{N}_{R} \neq \overleftarrow{\mathcal{N}_{L}}$ (where $\overleftarrow{\mathcal{N}}$ is the spatial inverse of the node $\mathcal{N}$ ), and that we want to build ${ }^{I} G_{\boldsymbol{r}}^{(k)}$ by erasing the right half of $G_{r}^{(k)}$. We then represent arc by drawing a heavy line (to distinguish the JA from the non-JAs) from $N_{L}$ to $\overleftarrow{\mathcal{N}_{R}}$ (which is in the left half). Furthermore, in ' $G_{\boldsymbol{r}}^{(k)}$, arc is a 'sink': 

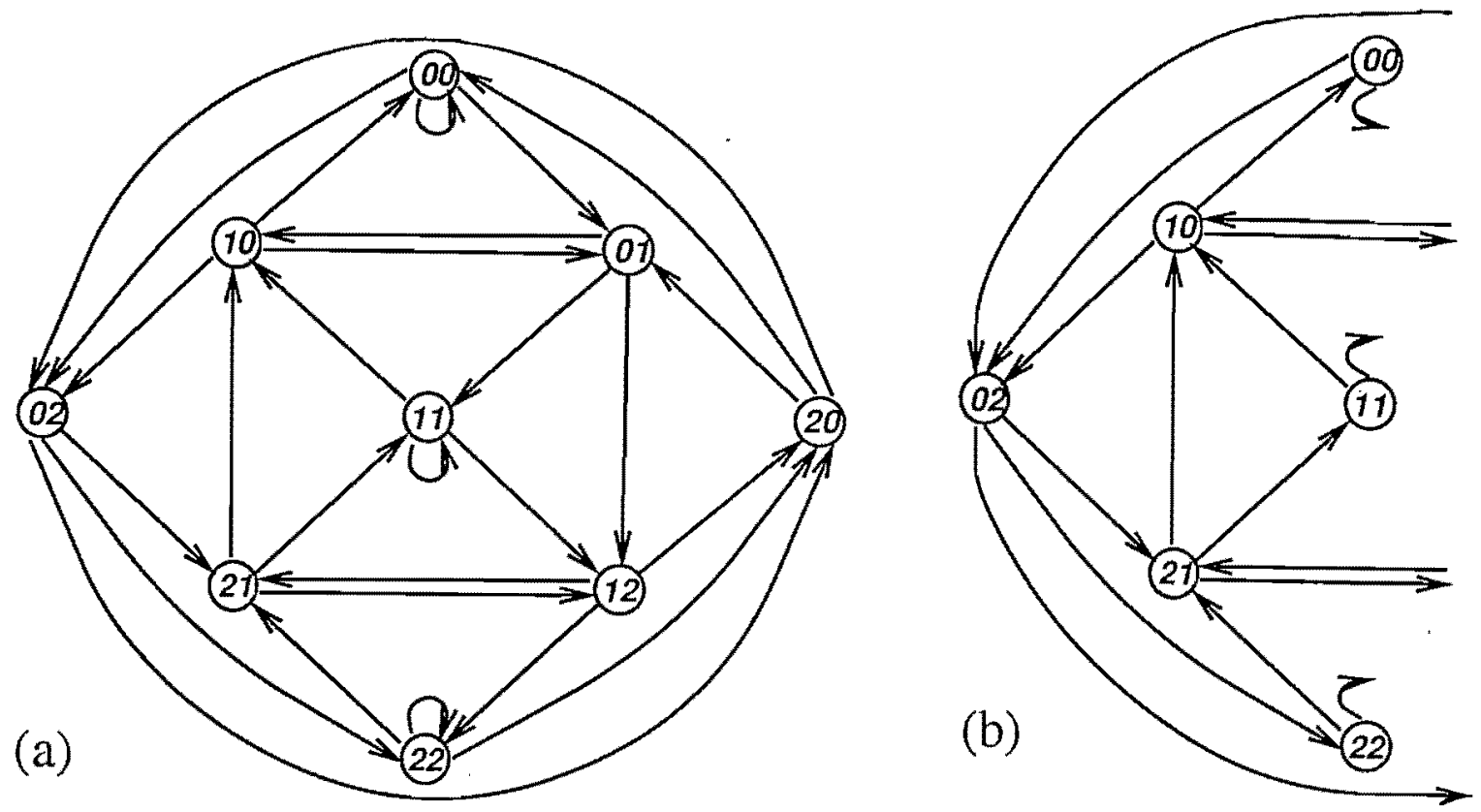

FIG. 3. (a) $G_{2}^{(3)}$; (b) ${ }^{I} G_{2}^{(3)}$. See text for a definition of cycles and simple cycles of ${ }^{I} G_{r}^{(k)}$.

it leaves both $N_{L}$ and $\overleftarrow{\mathcal{N}_{R}}$. (Had arc entered $N_{L}$, it would be a 'source': it would also enter $\overleftarrow{\mathcal{N}_{R}}$.) The resulting construction is shown in Fig, 4, using $G_{5}^{(2)}$ as an example.

This new feature of ${ }^{I} G_{r}^{(k)}$ requires yet further broadening of our definition of a cycle. The rule is, two paths flowing (in the same direction) from a single source to a single sink also constitute a cycle. We also allow the possibility that $\mathcal{I}$ can act as a source or sink. For bookkeeping, we label the paths leaving a source (and entering a sink) with distinct 'colors' (hence only two colors are needed). We allow further sources and/or sinks (ie, JAs) in each path-with the path changing color (and apparent direction) when crossing a JA-with the constraint that the colors must match at every node that is neither source nor sink.

Clearly, a type (i) (closed in ${ }^{I} G_{r}^{(k)}$ ) cycle which does not touch $\mathcal{I}$ must then include an even number of JAs, so that the path directions (colors) match everywhere away from the JAs. A type (ii) can include an odd number, in which case it uses $\mathcal{I}$ either as a source or as a sink.

Since sources and sinks in ${ }^{I} G_{\tau}^{(k)}$ merely amount to crossing $\mathcal{I}$ in $G_{\tau}^{(k)}$, it is perhaps clear that our specification of cycles of ${ }^{I} G_{\tau}^{(k)}$ will yield, upon 'unfolding' to $G_{\tau}^{(k)}$, cycles of the latter as well. 

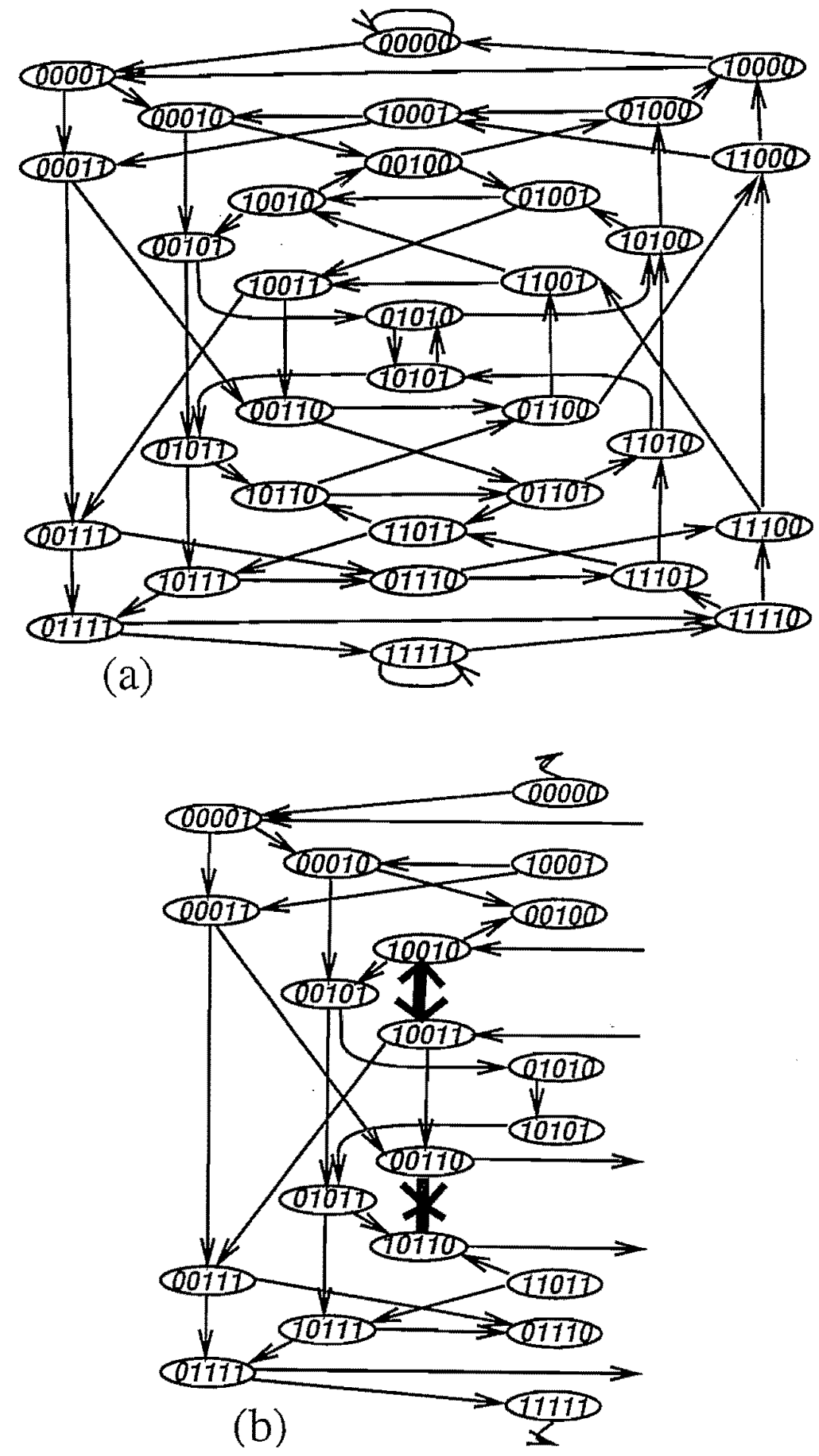

FIG. 4. (a) $G_{5}^{(2)}$; (b) ${ }^{I} G_{5}^{(2)}$. Note the appearance of the joining arcs (JAs-asymmetric arcs crossing the central line of symmetry) in both (a) and (b); in the latter, JAs appear as heavy lines with double arrowheads. 
This completes our construction of ${ }^{I} G_{r}^{(k)}$, including the definition of its cycles. However we still need an appropriate definition of a simple cycle of the graph ${ }^{I} G_{r}^{(k)}$. We will classify the SCs of ${ }^{I} G_{r}^{(k)}$ into four topological types. First we consider those that use no sources or sinks, ie, a single color. Such SCs include: (1) A simple closed loop in ${ }^{I} G_{r}^{(k)}$, which does not touch $\mathcal{I}$ [Fig. 5(a)]. (2) Same as (1), except one node (and only one) is on $\mathcal{I}$ [Fig. 5(b)]. (3) A non-self-intersecting path from one node on $\mathcal{I}$ to another [Fig. 5(c)].

We now consider SCs using one or more sources/sinks. We can add an even number of JAs to a type (1), and will include the resulting SCs in type (1). A type (2) can add an even number of JAs by having only one color at $\mathcal{I}$, or an odd number by having $\mathcal{I}$ serve as source or sink. The same holds true for type (3).

The final step is to consider allowing paths of differing color to touch. Such a rule makes sense, since, when unfolded, such paths are on opposite sides of $G_{r}^{(k)}$ and so do not intersect in $G_{r}^{(k)}$. Assume two paths of different color ('red' and 'blue') touch in a contiguous sequence of nodes. Call this part a 'red/blue contact' or RBC. We allow only a single RBC in a SC of ${ }^{I} G_{r}^{(k)}$; furthermore, there are constraints on how the paths terminate (meet) at each end. The red and blue paths may diverge before annihilating at a JA in ${ }^{I} G_{\boldsymbol{r}}^{(k)}$ _- but, once diverged, may not recontact, by the 'one- $\mathrm{RBC}$ ' rule. In contrast, if, at either end, the two annihilate at $\mathcal{I}$, then no separation of the two is allowed; that is, the RBC must include $\mathcal{I}$.

How do these new possibilities alter our topological types? We add a single RBC to type (1), 'pinching' it somewhere such that red meets blue. We call the result type (4); it is an RBC terminated by JAs at both ends [Fig. 5(d)]. By the above rules, we can only pinch a type (2) starting from $\mathcal{I}$; the result is still a type (2), but with an $\mathrm{RBC}$ 'neck' of more than one node [Fig. 5(e)]. Finally, we can either pinch a type (3) not at all, or everywhere-however a type (3) that is all $\mathrm{RBC}$ is equivalent to one which has no $\mathrm{RBC}$. Thus, the addition of RBCs augments our list of topological types of SCs by one.

Our specification of SCs of ${ }^{I} G_{r}^{(k)}$ is considerably more involved than that for ${ }^{s} G_{r}^{(k)}$. This is because of two complications: the treatment of $\mathcal{I}$ as a node, and the use of two colors, 


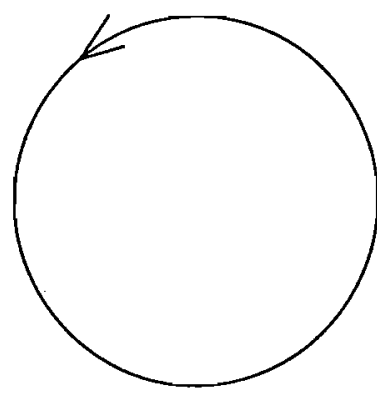

(a)

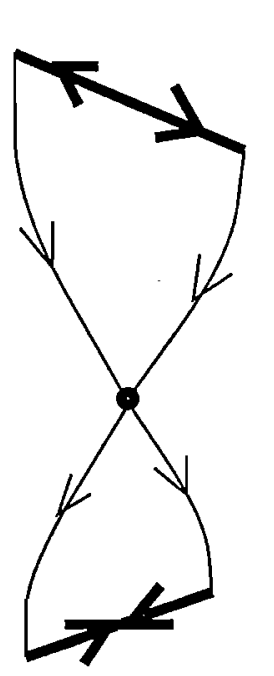

(d)
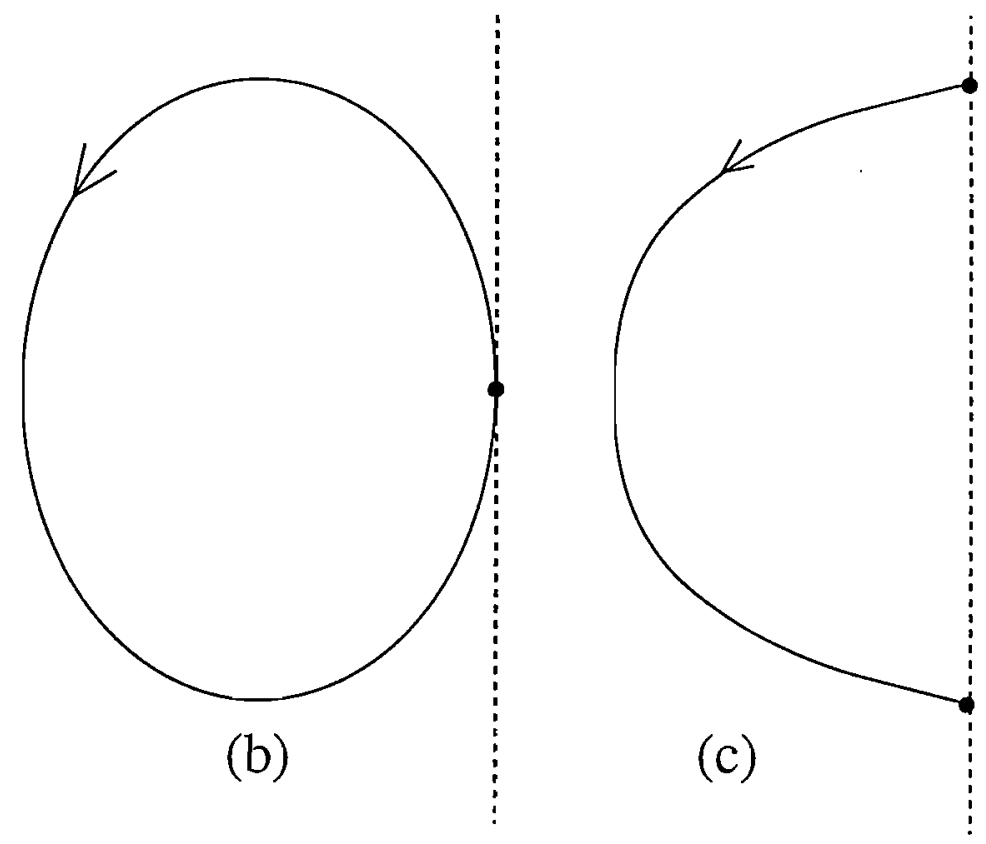

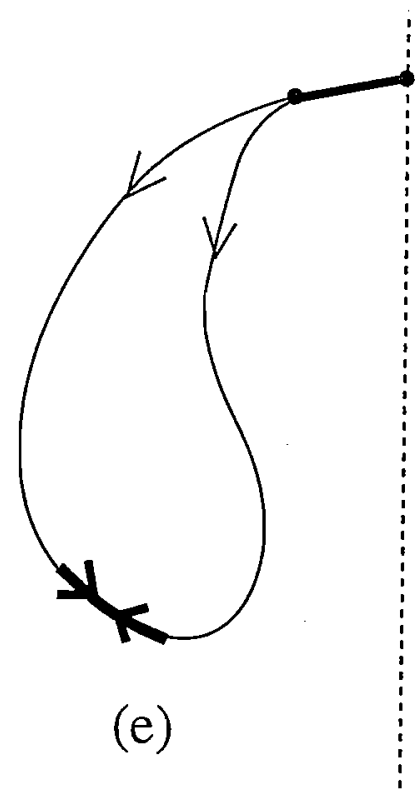

FIG. 5. The SCs of ${ }^{I} G_{r}^{(k)}$, presented in shematic form as four topological types. (a) A type (1) SC. (b) A type (2) touches $\mathcal{I}$ at a single node. (c) A type (3). (d) A type (4) SC. This is an example of a SC consisting of two paths ('red' and 'blue') running from source to sink. The red and blue touch at one or more contiguous nodes (an RBC, marked by a single large dot) in the center. (e) A type (2) which uses $\mathcal{I}$ as a source, and has an RBC (the upper heavy line) which includes $\mathcal{I}$. 
with the consequent possibility of RBCs. However, our overall criterion for a SC of ${ }^{I} G_{\boldsymbol{r}}^{(k)}$ is the same as that for a SC of ${ }^{S} G_{r}^{(k)}$, or of $G_{*}^{(k)}:$ a $S C$ of ${ }^{I} G_{r}^{(k)}$ is one for which there is no ambiguity as to which arcs of ${ }^{I} G_{r}^{(k)}$ are to be traversed. As we will see, it is this 'no-ambiguity rule' (implicit in the above detailed rules) which prevents SCs of ${ }^{I} G_{?}^{(k)}$ from decomposing.

Our definition of ${ }^{I} G_{r}^{(k)}$ and its SCs is complete. We now proceed to show that the SCs of ${ }^{I} G_{r}^{(k)}$ are the GSs of $H^{I}$.

$\underline{S C s}$ of ${ }^{I} G=G S s$ of $H^{I}$ :- First we show that non-SCs of ${ }^{I} G_{r}^{(k)}$ are not GSs of $H^{I}$. Our specification of SCs of ${ }^{I} G_{\tau}^{(k)}$ as a list of possibilities amounts to forbidding three things: (a) self-intersection with the same color (b) more than one RBC, or (c) improper termination of a single RBC. We next consider violating these three prohibitions, in order. Our goal is to show that violation of any of (a)-(c) means the resulting cycle is not a GS of $H^{I}$.

(a) Assume self-intersection by paths of the same color in a cycle of ${ }^{I} G_{\tau}^{(k)}$. We recall that cycles of ${ }^{I} G_{\boldsymbol{r}}^{(k)}$ in general give rise to pairs of cycles of $G_{\boldsymbol{r}}^{(k)}$, with each half of the pair related by $I$. Clearly, a necessary condition for cycle of ${ }^{I} G_{\tau}^{(k)}$ to be a GS of $H^{I}$ is that the resulting pair in $G_{\tau}^{(k)}$ be a pair of SCs (related by $I$ ). The pair must also not decompose, in the sense described in Section A above (on spin inversion symmetry).

Most cases of violation of (a) will give pairs in $G$ which are not SCs and so fail the first test. An exception is a same-color contact occurring on $\mathcal{I}$, as shown in Fig. 6(a). (This represents a same-color contact because $\mathcal{I}$ is a single 'node', which here is visited twice.) This type of cycle unfolds in $G$ as shown in Fig. 6(b), and decomposes to two symmetric cycles in a manner similar to that seen in the case of $S$ symmetry. A variation on this violation, which also represents (and also decomposes to) a pair of type (3) SCs in ${ }^{I} G_{r}^{(k)}$, is shown shematically in Figs. 6(c)-(d).

(b) Consider a cycle of ${ }^{I} G_{r}^{(k)}$ with two RBCs. We suppose that the two RBCs, which are connected by a red/blue 'bubble', are terminated by a JA at one end [a 'stirrup'—cf Fig. 7(a)] and by $\mathcal{I}$ at the other. The resulting set of $\operatorname{arcs}$ in $G$ [Fig. 7(b)] may be viewed, with some care, as a symmetry-related pair of SCs, of intensive energy $(a+b+c) /(\ell+m+n)$. However, 


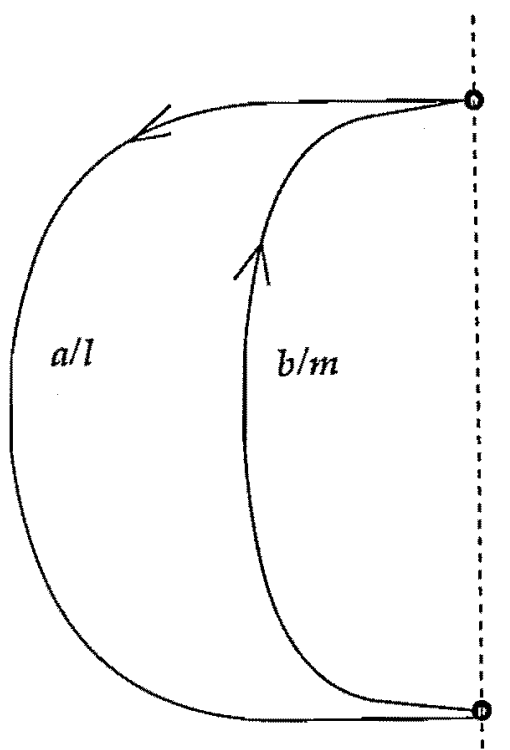

(a)

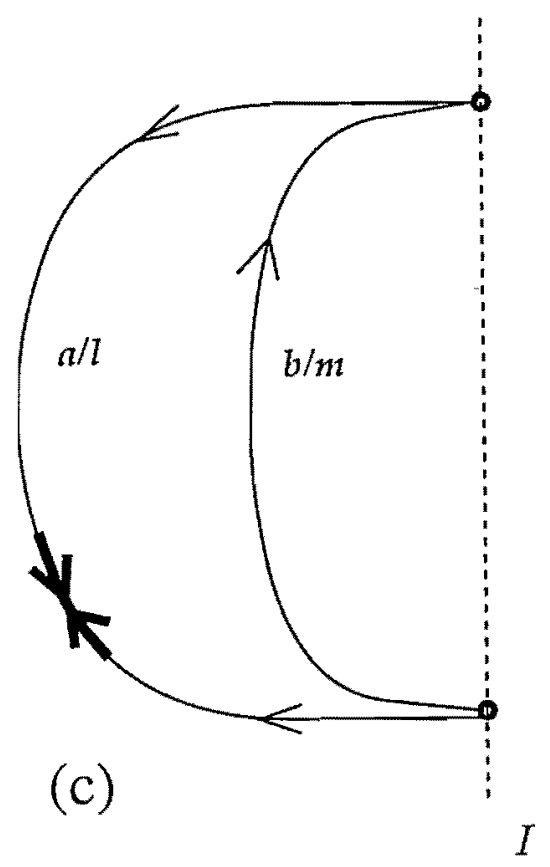

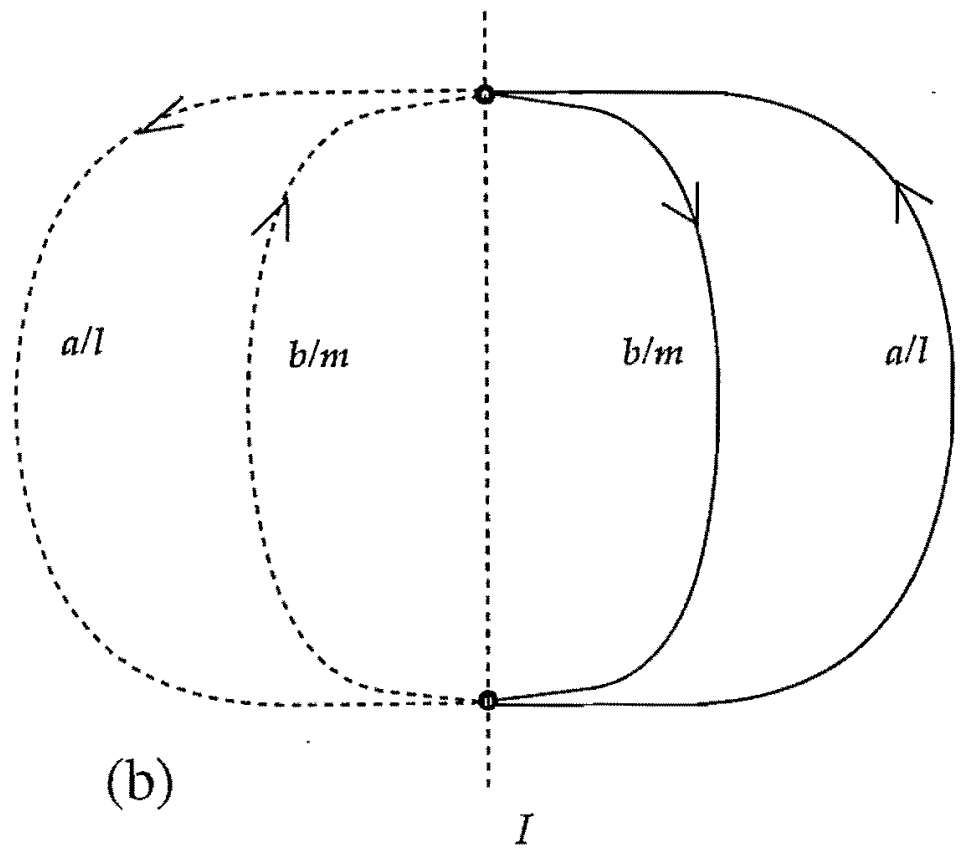

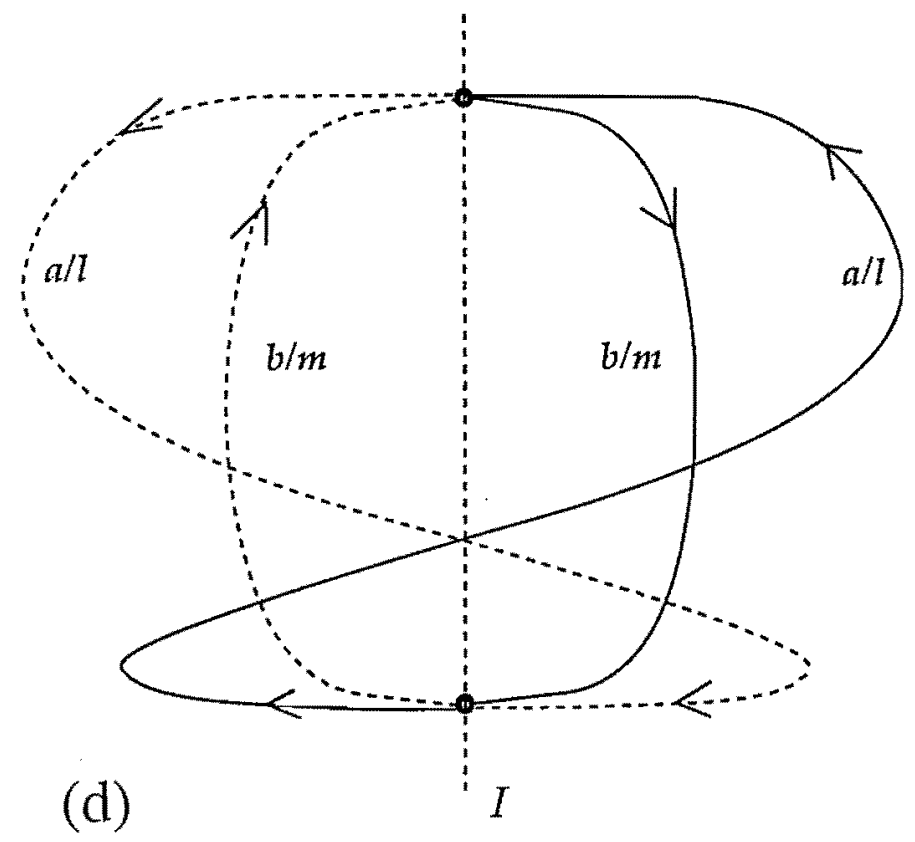

FIG. 6. (a) A non-SC of ${ }^{I} G_{r}^{(k)}$ (schematic). The schematic shared 'nodes' (large dots) on $\mathcal{I}$ (vertical dashed line) may be either $I$-symmetric arcs or nodes. (b) The appearance of (a) in $G_{r}^{(k)}$; compare Fig. 2(b). Again the asymmetric SCs, of weight $(a+b) /(\ell+m)$, are never ground states when $H$ is $I$-symmetric. Note that changing the relative direction of the two pieces in (a) [or in (c)] leaves the logic unchanged. (c),(d): Same as (a),(b), except there is a JA in part of the cycle. 
because the no-ambiguity rule is violated, this pair also defines two other (symmetric) SCs of (intensive) weight $(2 a+c) /(2 \ell+n)$ and $(2 b+c) /(2 m+n)$, respectively. Now assume the pair represented by Fig. 7(a) is a GS, so that

$$
\frac{a+b+c}{\ell+m+n}<\frac{2 a+c}{2 \ell+n} \quad \text { and } \quad \frac{a+b+c}{\ell+m+n}<\frac{2 b+c}{2 m+n} .
$$

If we multiply out each of these inequalities (assuming $\ell, m$, and $n$ positive) we obtain a contradiction. Hence the non-SC in Fig. 7(a) cannot be a GS; it decomposes [Fig. 7(c),(d)]. The same arithmetic and conclusion, with slightly different pictures, applies when the pair of RBCs is terminated by stirrup + stirrup, or by $\mathcal{I}+\mathcal{I}$. Therefore, any SC of $G_{r}^{(k)}$ which is not a SC of ${ }^{I} G_{r}^{(k)}$ by virtue of violation of (b) is not a GS of $H^{I}$.

(c) We finally consider improper termination of an $\mathrm{RBC}$, by divergence before termination on $\mathcal{I}$. It is easily seen by simple sketches that, regardless of the other termination of the RBC (stirrup, or properly on $\mathcal{I}$ ), the resulting pair are not SCs of $G$ and so fail to be GSs of $H^{I}$.

Summarizing the above, we find that, for any conceivable violation of the rules for a SC of ${ }^{I} G_{r}^{(k)}$, the resulting cycle is not a GS of $H^{I}$. Turning this around, we find that all GSs of $H^{I}$ are $\mathrm{SCs}$ of ${ }^{I} G$.

We note in passing that Morita ${ }^{9}$ previously obtained results which foreshadow ours. $\mathrm{He}$ assumed $I$ symmetry of $H$, and found necessary conditions for cycles of $G$ to be GSs of $H^{I}$. In our language, he found that such allowed cycles must touch $\mathcal{I}$ no times [type (1) and type (4)], or once [type (2)] or if twice, must be $I$-symmetric [type (3)]. These conditions, which are satisfied by our SCs, are not sufficient to ensure a GS. Specifically, Morita's rules encapsulate our rule (a) (no same-color contact), but fail to capture (b) and (c) (which give constraints on RBCs).

Finally, we need the converse: that all SCs of ${ }^{I} G_{\tau}^{(k)}$ are GSs of $H^{I}$. Here the argument is essentially unchanged from that for the case of $S$ symmetry: densities (arcs) related by $I$ are considered not distinct, and it readily follows that SCs of ${ }^{I} G_{r}^{(k)}$ are vertices of ${ }^{I} P_{r}^{(k)}$ - the intersection $^{7}$ of $P_{r}^{(k)}$ with the $I$-invariant subspace. 

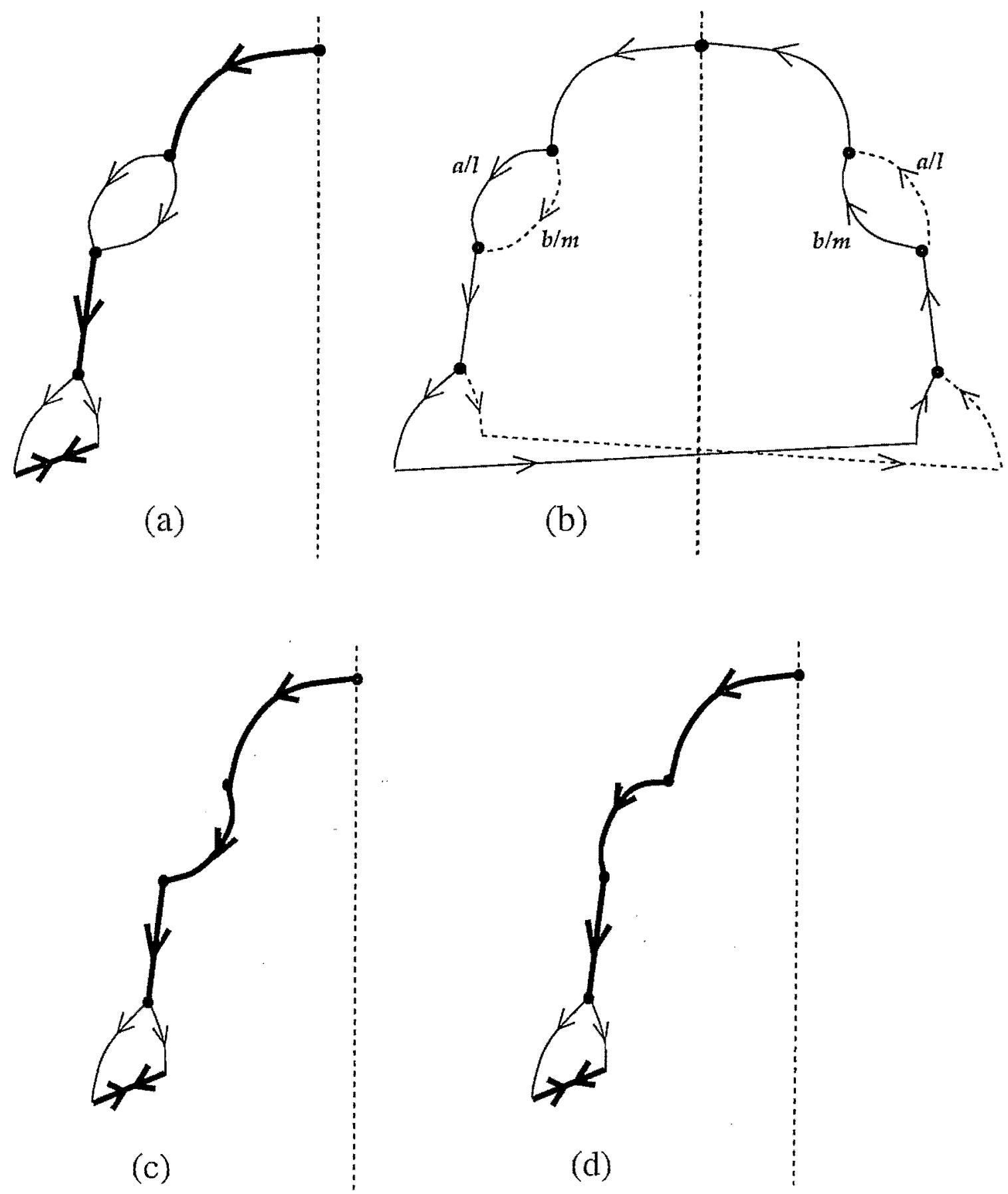

FIG. 7. (a) A cycle of ${ }^{I} G_{r}^{(k)}$ with two RBCs. (b) We view (as always) the cycle of ${ }^{I} G_{r}^{(k)}[$ in (a)] as representing two symmetry-related cycles in $G_{r}^{(k)}$, shown here. Where the two cycles differ, one is dashed and the other solid. We take the net intensive weight of all arcs outside the 'bubbles' to be $c / n$-which is the same for both cycles by symmetry. Each of the two cycles is a SC of $G_{r}^{(k)}$ but neither is a GS of $H^{I}$ (see text). (c), (d) The decomposition of the cycle of (a) to two [type (2)] SCs of ${ }^{I} G_{r}^{(k)}$, each with a single long RBC. Both (c) and (d) represent GSs of $H^{I}$. 
We are now ready to seek D-pairs in ${ }^{I} G_{r}^{(k)}$. To this end we can use our established classification of the SCs of ${ }^{I} G_{r}^{(k)}$ into four types. A type (1) SC gives rise to a pair of nonintersecting SCs in $G$. Such a GS thus represents spontaneous breaking of $I$ symmetry (SSB), but not a D-pair. All type (2) SCs represent D-pairs in $G_{r}^{(k)}$, sharing one or more nodes which straddle $\mathcal{I}$. Type (3) SCs of ${ }^{I} G$ map to symmetric SCs of $G$, and so are not D-pairs. Finally, type (4) SCs give D-pairs sharing one or more nodes which do not touch I.

Hence our search for D-pairs is a search for SCs of type (2) or (4) in ${ }^{I} G$. We first consider $r=1$. For this case, all nodes are on $\mathcal{I}$. Hence all SCs of ${ }^{I} G_{1}^{(k)}$ are of type (3), and there are no D-pairs.

We now use the fact that (in this section) $k>2$, and consider $r>1$, in a recursive rep. In this case, there are always SCs of $G$ whose nodes are of the form $L \ldots L S$ (where ... is a string of $L$ 's). Specifically, a cycle of the form $L^{j} S$ (nodes) in $G_{r}^{(k)}$ may be built, in a recursive rep, from a cycle in $G_{1}^{(k)}$ whose arcs (all nodes being $S$ ) take the form $L^{j-(r-2)} S^{r-1}$. The $I$-partner of such a SC in $G_{r}^{(k)}$ is then of the form $R^{j} S$. A pair of SCs of $G$, of this form, is a type (2) SC of ${ }^{I} G$, with a single node (and a single color) at $\mathcal{I}$; and the pair is a $D$-pair. For example, in $G_{2}^{(3)}$, one can build $L L L S$ (nodes) SCs from $L L L S$ (arcs) of $G_{1}^{(3)}$; these form D-pairs in $G_{2}^{(3)}$ with their partners RRRS. There are three such pairs- $(2210) /(0122)$, $(2110) /(0112)$, and $(2100) /(0012)$ - as may be seen in Fig. 3(b). For larger $k$ and/or $r$, the number of such D-pairs increases (and other types appear). Hence we find that

Disordered GSs occur in the case of I symmetry for any $k$-state problem with $k \geq 3$ and $r \geq 2$. 


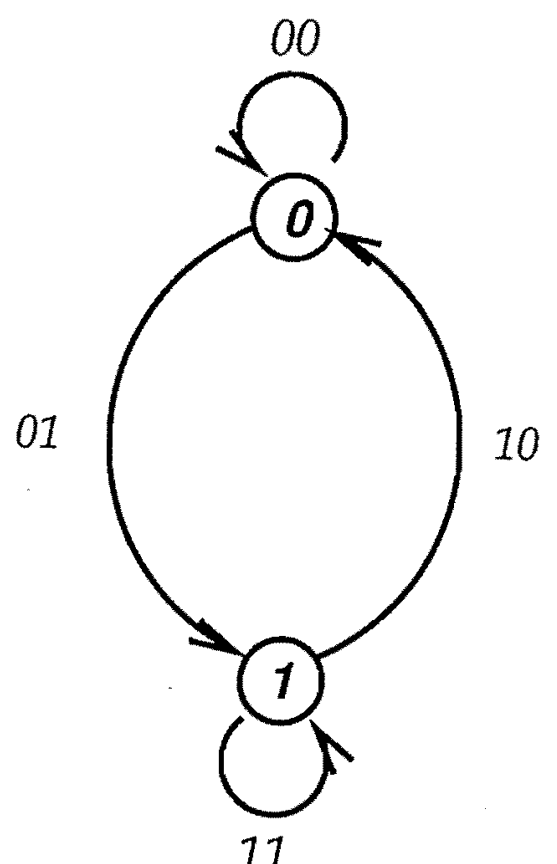

FIG. 8. $G_{1}^{(2)}$. The choice of handedness for the two nonsymmetric arcs of $G_{1}^{(k)}$ is really no choice, since the two must be opposite. Hence, until JAs occur at $r=5$, there is effectively only one recursive rep for $k=2$.

\section{3. $k=2$}

The Ising case $(k=2)$ has some special properties. In particular, $G_{1}^{(2)}$ (Fig. 8) has a single $L$ arc and a single $R$ arc. This makes it impossible to make any cycle in $G_{1}^{(2)}$ in which an $L$ arc follows another $L$ (similarly with $R$ ), so that our above argument-by-construction, using a recursive rep for $k>2$, fails for $k=2$. In fact, even with multiple $S$ arcs [(11) or $(00)$ ] after a given $L$, it is clear that the first non- $S$ arc after the $L$ must be an $R$. In other words, all cycles of $G_{1}^{(2)}$, of any length, are of the form

$$
\ldots R \ldots L \ldots R \ldots L \ldots R \ldots L \ldots
$$

where ... is a string of $S$ 's of any length.

From this we can deduce another property which is peculiar to the Ising case in the recursive rep. The property (8) implies that every cycle of $G_{1}^{(2)}$ [except the two ferromagnetic cycles $(1)$ and $(0)]$ crosses $\mathcal{I}$ an even $(\geq 2)$ number of times. One can easily show that the 
same is true for $G_{r}^{(2)}$, for any $r \leq 5$, in the recursive rep. ${ }^{10}$ We then note the following: (i) the recursive rep is a minimal rep for $r \leq 5$; (ii) in the recursive rep, $G_{\tau}^{(2)}$ has $n_{J}=0$ for $r<5$, and $n_{J}>0$ for $r \geq 5$; (iii) hence $G_{r}^{(2)}$ is $\mathcal{I}$-disjoint for $r<5$ and non- $\mathcal{I}$-disjoint for $r=5$. (These statements may be verified by explicit construction of the recursive rep.)

Our 'even-crossing rule', plus (i)-(iii) of the previous paragraph, suffice to ensure that there are neither D-pairs nor SSB in $G_{r}^{(2)}$ for $r<5$, as follows: Type (1) cannot occur in the recursive rep due to even-crossing plus $\mathcal{I}$-disjointness; hence it cannot occur in any rep. Type (2) is similarly ruled out. Type (4) requires JAs; this type is ruled out by (iii) for $r<5$. Thus all SCs of ${ }^{I} G_{\boldsymbol{r}}^{(2)}$, for $r<5$, are of type $(3)$-symmetric cycles of $G$-and hence represent neither D-pairs nor SSB.

We next consider the case $r=5$. Here we find four JAs (Fig. 4) in the recursive rep (which is still minimal). The presence of these JAs is sufficient to allow three of the four types of SCs in ${ }^{I} G_{5}^{(2)}$. For example, a type (2) SC, and its unfolding, are shown in Fig. 9. An example (the only one) of SSB in $G_{5}^{(2)}$ is the pair (101100) and its inverse (001101); this pair gives a type (1) SC of ${ }^{I} G_{5}^{(2)}$ which uses both of its JAs.

Given that $G_{r}^{(2)}$ is non- $\mathcal{I}$-disjoint, one can show that $G_{\tau+1}^{(2)}$ is also. (In fact, this is true for any $k{ }^{11}$ ) Furthermore, even in a minimal rep, $n_{J}$ increases with increasing $r$. The result is that types (1)-(3) occur for all $r \geq 5$. We also find (aided by a computer search) that type (4) D-pairs ${ }^{12}$ occur for $r \geq 7$. Hence we find that

Both SSB and disordered, degenerate GSs occur in the Ising problem with I symmetry for $r \geq 5$. Neither occurs for $r<5$.

\section{C. $S I$ symmetry}

$S I$ symmetry may be handled very much like $I$. (The symmetry line in this case is a horizontal line $\mathcal{S I}$ through the center of the graphs.) Here we just note the conclusions. The results for $k=2$ are the same (an 'even-crossing rule' in the recursive rep, non- $\mathcal{S I}$ disjoint for $r \geq 5$, and disordered GSs for $r \geq 5$ ). $G_{\tau}^{(3)}$ is non-SI-disjoint for $r \geq 3 ; G_{r}^{(k)}$ 

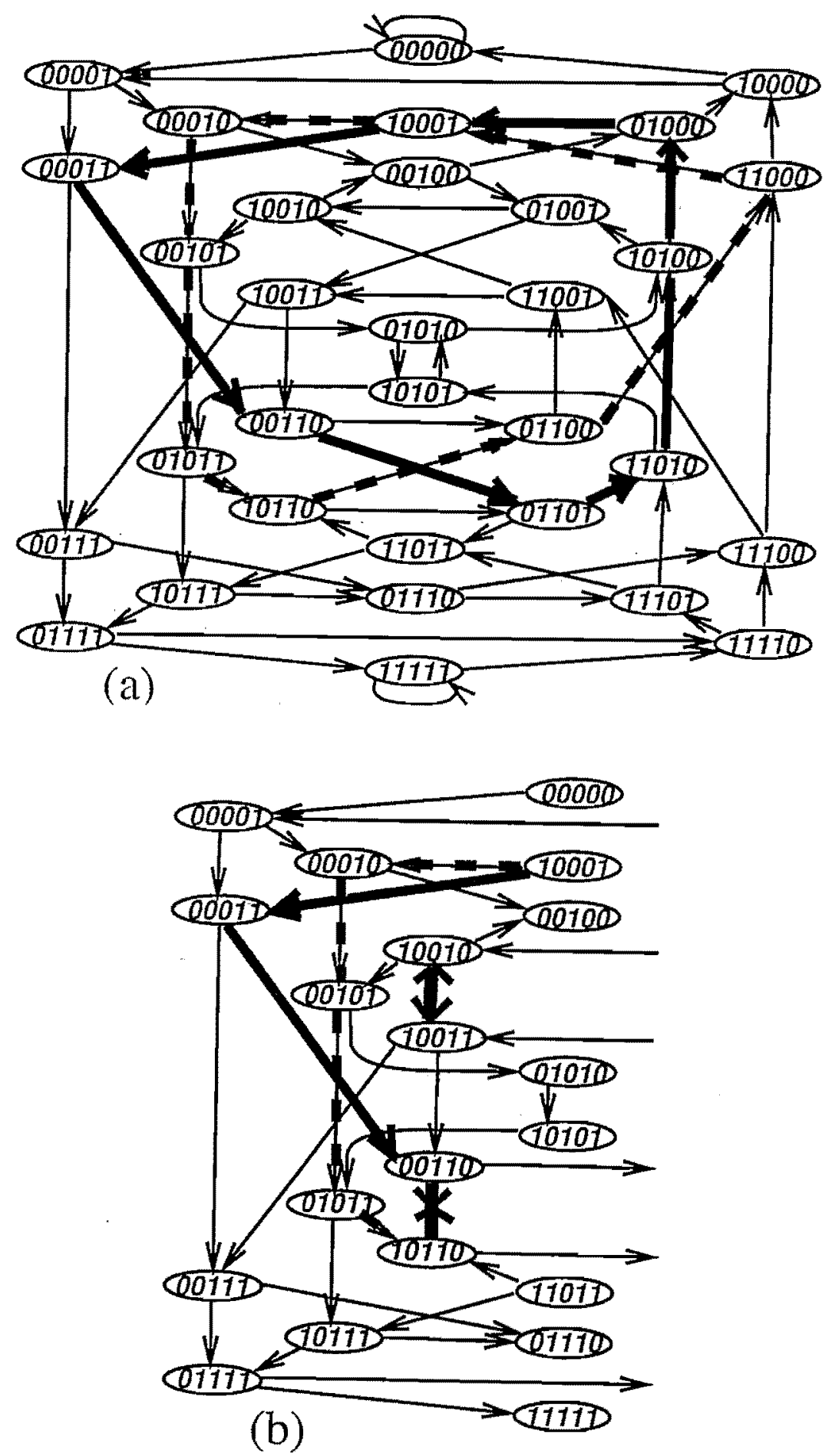

FIG. 9. (a) A D-pair (heavy solid, and heavy dashed lines) in $G_{5}^{(2)}$. Such a pair of cycles gives rise to a degenerate set of configurations, including disordered ones, which are GSs over a finite volume of coupling-parameter space. (b) The appearance of the two SCs of (a) as a type (2) SC in ${ }^{I} G_{5}^{(2)}$. The two 'legs' going from the symmetry line $\mathcal{I}$ to the JA have different 'colors' (here represented by solid vs. dashed lines). 
is non- $\mathcal{S I}$-disjoint for any $k \geq 4$ and $r \geq 1$. There are disordered GSs for any $k \geq 3$ and $r \geq 1$.

\section{D. $(S+I)$ symmetry}

We finally consider the case where both $S$ and $I$ are good symmetries of $H$. We construct the graph ${ }^{s+I} G_{r}^{(k)}$ by applying $I$ symmetry to ${ }^{s} G_{r}^{(k)}$ (erasing half of it, and correcting for JAs). We can then use arguments like those above to show that the $\mathrm{SCs}$ of ${ }^{S+I} G_{r}^{(k)}$ (defined similarly to those of ${ }^{I} G_{r}^{(k)}$ ) are the GSs of $H^{S+I}$. (In particular, the same arguments, used in Section B to eliminate SCs of $G_{r}^{(k)}$ which are not SCs of ${ }^{I} G_{\tau}^{(k)}$, may be used to eliminate SCs of ${ }^{s} G_{r}^{(k)}$ which are not SCs of ${ }^{S+I} G_{r}^{(k)}$; and the usual argument shows that all SCs of ${ }^{S+I} G_{r}^{(k)}$ are GSs of $H^{S+I}$.)

For what values of $r$ and $k$ do we find disordered GSs of $H^{S+I}$ ? Again we just give our conclusions here. Combining the two symmetries eliminates some D-pairs, and creates others. With one exception, however, we find that, wherever (in $r$ and $k$ - see Table I below) $S$ or $I$ alone gives disordered GSs, the combination $S+I$ also gives disordered GSs. The exception is $G_{5}^{(2)}$. Here we find (as noted above) that ${ }^{s} G_{5}^{(2)} \sim G_{4}^{(2)}$. Since the latter graph has no D-pairs, application of $I$ to ${ }^{s} G_{5}^{(2)}$ gives a graph ${ }^{s+I} G_{5}^{(2)} \sim{ }^{I} G_{4}^{(2)}$ which also has no D-pairs. We note finally that $S+I$ does not give disordered GSs where neither $S$ nor $I$ does.

\section{DISCUSSION, EXAMPLE, AND SUMMARY}

We summarize our findings in Table I. Each $X$ entry means that there are D-pairs in $G_{r}^{(k)}$, arising from the the appropriate types of SCs in ${ }^{x} G_{r}^{(k)}$. This in turn means that for the given $r$ and $k$, the corresponding $k$-state problem has degenerate, disordered GSs, arising from $X$ symmetry, over a finite region of coupling-parameter space, without any fine tuning (beyond that coming from the symmetry). 
TABLE I. One-dimensional $k$-state models which allow for disordered ground states, with no fine tuning of coupling parameters other than that demanded by symmetry. Rows are indexed by the number of states $k$, and columns by the range $r$ (in lattice constants) of the interactions. An entry is made whenever spin inversion $(S)$ or spatial inversion $(I)$ symmetry gives rise to one or more pairs of degenerate ground states, with zero surface tension between the two states of the pair. It is this combination (degeneracy plus zero surface tension) which allows for disordered ground states.

\begin{tabular}{rrrrrrrr}
\hline \hline & $\mathrm{r}=1$ & 2 & 3 & 4 & 5 & 6 & $\ldots$ \\
\hline$k=2$ & & & & & $I$ & $I$ & $\ldots$ \\
3 & $S, I$ & $S, I$ & $S, I$ & $S, I$ & $S, I$ & $\ldots$ \\
5 & & $I$ & $I$ & $I$ & $I$ & $I$ & $\ldots$ \\
6 & $S$ & $S, I$ & $S, I$ & $S, I$ & $S, I$ & $S, I$ & $\ldots$ \\
$\vdots$ & & $I$ & $I$ & $I$ & $I$ & $I$ & $\ldots$ \\
\hline \hline
\end{tabular}




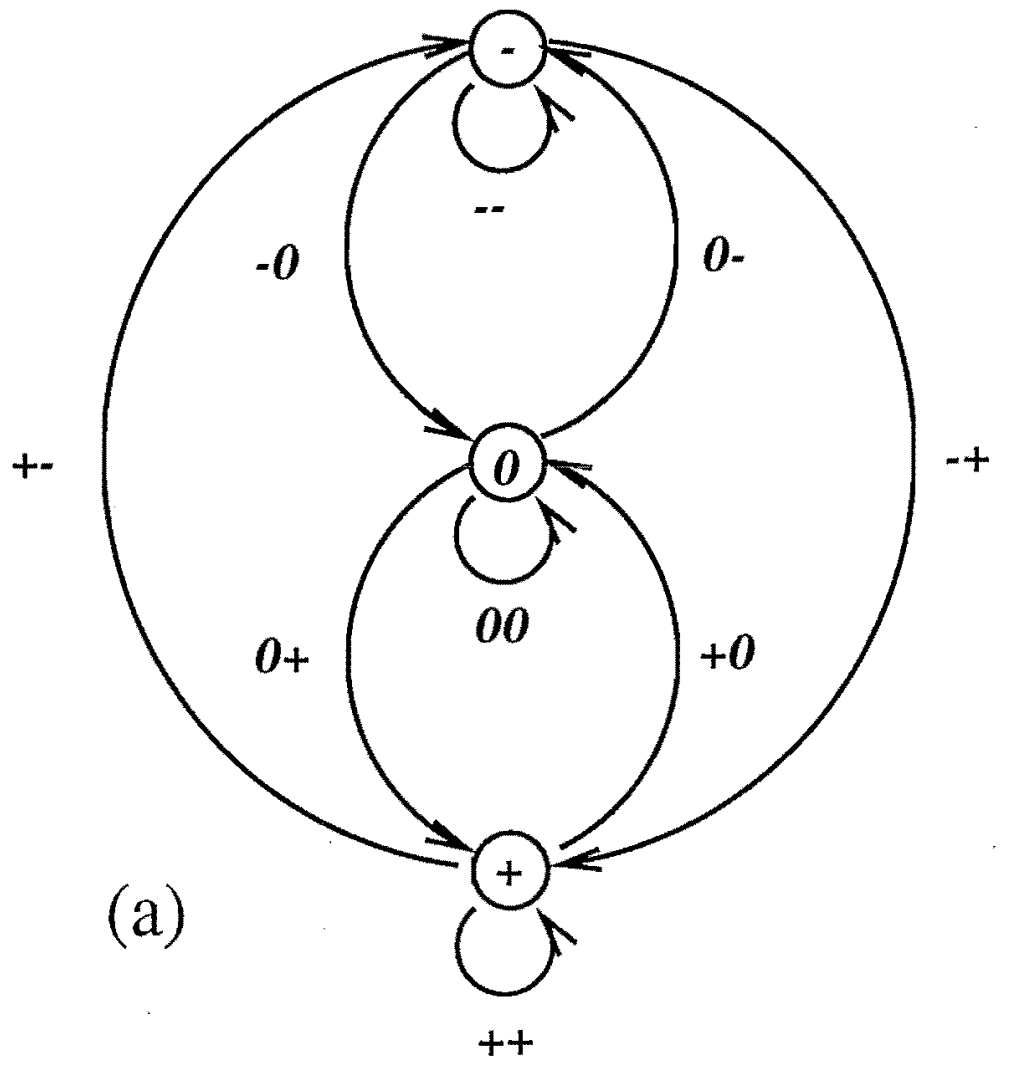

(b)

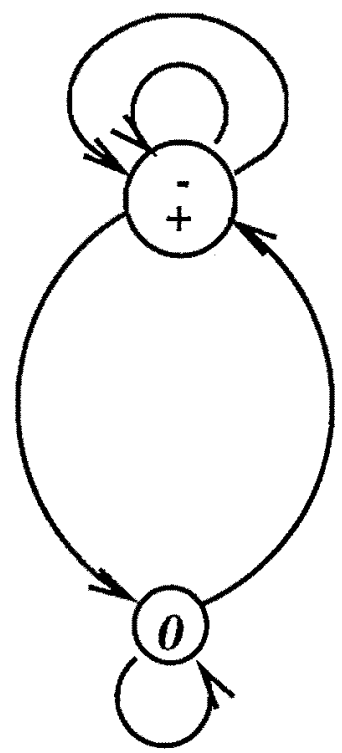

FIG. 10. (a) $G_{1}^{(3)}$; (b) $s_{G_{1}^{(3)}}$. In the latter, a pair of energetically distinct arcs connects the node $-/+$ to itself; in general, ${ }^{s} G_{r}^{(k)}$ has parallel arcs such as these for $k>2$, and so is not a de Bruijn graph.

We give here a simple example to illustrate the above (sometimes rather complicated) logic. The simplest entry from Table $I$ is $k=3, r=1$. We give both $G_{1}^{(3)}$ and ${ }^{s} G_{1}^{(3)}$ in Fig. 10; for convenience, we let the three states [formally labelled $(0,1,2)$ ] take the values +1 , 0 , and -1 (so that $S \sigma=-\sigma$ ). The dimension of $H$ is $d=6$; the independent correlations are $s_{1}=\left\langle\sigma^{2}\right\rangle, s_{2}=\left\langle\sigma_{i} \sigma_{i+1}\right\rangle, s_{3}=\left\langle\sigma_{i}^{2} \sigma_{i+1}^{2}\right\rangle, s_{4}=\langle\sigma\rangle, s_{5}=\left\langle\sigma_{i}^{2} \sigma_{i+1}\right\rangle$, and $s_{6}=\left\langle\sigma_{i} \sigma_{i+1}^{2}\right\rangle$. We then write the per-spin Hamiltonian, for an infinite chain, as $\mathcal{H}=-\mathbf{J} \cdot \mathbf{s}$.

Since none of the arcs in $G_{1}^{(3)}$ are constrained by either flow or symmetry, they represent 9 distinct densities, giving the 9 faces of the polytope $P_{1}^{(3)}$ which lives in 6-dimensional Euclidean space. (We will not give the relationships between the densities and the correlations here; they are readily generalized from those given by Teubner ${ }^{2}$ for $k=2$.)

Now, guided by Table I, we apply $S$ symmetry, and seek disordered GSs. This means 
$J_{4}=J_{5}=J_{6}=0$ in $\mathcal{H}$; hence the polyhedron ${ }^{s} P_{1}^{(3)}$ is the three-dimensional 'slice' of $P_{1}^{(3)}$ given by $s_{4}=s_{5}=s_{6}=0$. The five $\operatorname{arcs}$ of ${ }^{s} G_{1}^{(3)}$ give rise to five densities: $n_{-0}=n_{+0} \equiv n_{x 0}$; $n_{0-}=n_{0+} \equiv n_{0 x} ; n_{+-}=n_{-+} \equiv n_{x x}^{(1)} ; n_{++}=n_{--} \equiv n_{x x}^{(2)} ;$ and $n_{00}$. However only four of these are distinct, since the structure of ${ }^{S} G_{1}^{(3)}$ constrains $n_{0 x}=n_{x 0}$ by conservation of flow. Hence ${ }^{s} P_{1}^{(3)}$ is a polyhedron in $3 \mathrm{D}$ with four faces: it is a tetrahedron. The vertices of ${ }^{s} P_{1}^{(3)}$ are the four SCs of ${ }^{s} G_{1}^{(3)}:(0),(+)=(-),(+-)$, and $(0+)=(0-)$. The last is of course the D-pair, sharing the invariant node $\mathcal{N}^{*}=0$. Each of these SCs sets 3 of the 4 distinct densities to zero, hence shares $d^{S}=3$ of the $4\left(=n_{f}\right)$ faces of ${ }^{s} P_{1}^{(3)}$. In $\mathbf{s}$ coordinates, these vertices are, respectively, $(0,0,0),(1,1,1),(1,-1,1)$, and $(1 / 2,0,0)$. The SCs $(0-+)$ and $(0+-)$ of $G_{1}^{(3)}$ are not SCs of ${ }^{s} G_{1}^{(3)}$; they lie on the edge in ${ }^{s} P_{1}^{(3)}$ joining $(0+) /(0-)$ to (+-). This is a geometric version of decomposition (recall Fig. 2 and the associated discussion); one can verify graphically that $(0-+) /(0+-)$ decomposes to $(0+) /\left(0_{-}\right)$and $(+-)$.

The (single) D-pair for this case is the lowest-energy SC in the subvolume bounded by the planes $J_{1} / 2+J_{2}+J_{3}<0, J_{1} / 2-J_{2}+J_{3}<0$, and $J_{1}>0$. For illustration, a representative point in this subvolume is $\mathcal{H}=-\left\langle\sigma^{2}\right\rangle+\left\langle\sigma_{i}^{2} \sigma_{i+1}^{2}\right\rangle$-that is, $J_{1}=+1, J_{2}=0$, and $J_{3}=-1$.

It would be of some interest to find an analogous neighborhood for the smallest- $\boldsymbol{r}$ Ising $(k=2)$ problem with disordered GSs: $r=5$ for $I$ symmetry, and $r=6$ for $S+I$ symmetry. This task however is considerably more tedious than the above simple example; hence we do not attempt it here.

Table $I$ is striking in the near-ubiquity of its entries, which stands in strong contrast to the simple result of Radin and Schulman. ${ }^{1}$ Given that $I$ symmetry is ubiquitous as well, we might expect disordered GSs for a number of interesting problems. However, both the present results, and those of $\mathrm{RS}$, remain somewhat academic in the absence of a convincing physical application. It may, or may not, be the case that the regions of parameter space giving disordered GSs are in general too 'weird' (ie, unphysical) to be visited by physical problems. Only further work can answer this question. The answer is however of considerable interest since it has relevance to the Third Law of thermodynamics. ${ }^{13}$ 
We note finally that there is at least one physically-motivated problem, namely the problem of stacking polytypes in crystals, ${ }^{14}$ which should be well modelled by an effective Ising Hamiltonian with medium- $^{15}$ or long-range ${ }^{16}$ effective interactions between the stacking units (layers). Materials showing polytypism do show very-long-period and disordered structures, even down to low temperatures. It is possible-perhaps likely-that all these complex structures are metastable configurations, trapped at low temperature by a 'rugged' energy surface. However, given our present results (and the $S+I$ symmetry of the problem), we believe that the possibility that some of the disordered structures are ground states (in the more common sense of the term: minimal-energy configurations) cannot be ruled out $a$ priori; hence such a possibility deserves further study.

Acknowledgements.- We thank Larry Schulman and Charles Radin for helpful discussions of their results, Hal Frederickson for guidance to the literature of de Bruijn graphs, and Ken Stephenson for suggesting that we consider the graphs ${ }^{x} G_{r}^{(k)}$, and for helpful discussions of geometry. This work was supported in part by the U.S. Department of Energy through Contract No. DE-AC05-84OR21400 with Martin Marietta Energy Systems Inc. GSC acknowledges support from the NSF under Grant \# DMR-9413057. 


\section{REFERENCES}

${ }^{1}$ Charles Radin and Lawrence S. Schulman, Phys. Rev. Lett. 51, 621 (1983).

${ }^{2}$ Max Teubner, Physica A 169, 407 (1990).

${ }^{3}$ M. Bundaru, N. Angelescu, and G. Nenciu, Phys. Lett. A 43, 5 (1973).

${ }^{4}$ D.F. Robinson and L.R. Foulds, Digraphs: Theory and Techniques, Gordon and Breach (1980); R.G. Busacker and T.L. Saaty, Finite Graphs and Networks, McGraw-Hill (1965).

${ }^{5}$ See Harold Frederickson, Discr. Appl. Math. 37/38, 193 (1992), and references therein; S.W. Golomb, Shift Register Sequences, Holden-Day (1967).

${ }^{6}$ Harold Frederickson, SIAM Review, 24, 195 (1982).

${ }^{7}$ Strictly, the pertinent lower-dimensional object is the projection of $P_{\tau}^{(k)}$ onto the invariant hypersurface defined by the symmetry. However, one can show, using the symmetry and convexity properties of $P_{r}^{(k)}$, that the projection is the section.

${ }^{8}$ We are indebted to Jim Hanson for the following observation. In the language of computation theory, the graph $G_{\tau}^{(k)}$ is a finite-state transducer whose input is a string of spin values and whose output is a string of arc weights. Disregarding the input, $G_{r}^{(k)}$ is a deterministic, finite-state automaton or DFA. Given $S$ symmetry, $G_{r}^{(k)}$ is no longer minimal, and can be reduced to a minimal DFA (ie, one capable of producing the same output strings with fewer nodes) which is in fact ${ }^{s} G_{r}^{(k)}$. In contrast, $I$ symmetry in general leaves $G_{\tau}^{(k)}$ minimal; hence our unconventional approach to ${ }^{I} G_{r}^{(k)}$ (Section B). See, for example, Hopcroft, J.E., and J.D. Ullman, Introduction to Automata Theory, Languages, and Computation, Addison-Wesley (1979), Chapter 3.

${ }^{9}$ T. Morita, J. Phys. A 7, 1613 (1974).

${ }^{10}$ For $r>5$ there are multiple recursive reps (due to the appearance of JAs at $r=5$ ). We conjecture (but do not need for our argument) that the even-crossing property holds in 
all recursive reps, even for $r>5$.

${ }^{11}$ We find that $G_{r}^{(3)}$ is non- $\mathcal{I}$-disjoint for $r \geq 4$, and $G_{r}^{(k)}$ is non- $\mathcal{I}$-disjoint for any $k \geq 4$ and $r \geq 2$.

12 An example is the cycle $(0001001101000110111)$ and its inverse in $G_{7}^{(2)}$, which has a singlenode $\mathrm{RBC}$ in ${ }^{r} G_{7}^{(2)}$. For $k=3$, we find type (4) SCs for $r \geq 4$; and for $(k=4, r \geq 3)$, $(k=5, r \geq 2)$, and $(k=6, r \geq 2)$.

${ }^{13}$ Jacek Miȩkisz and Charles Radin, Mod. Phys. Lett. B 1, 61 (1987), and references therein.

${ }^{14}$ See, for example, Ajit Ram Verma and P. Krishna, Polymorphism and Polytypism in Crystals, John Wiley \& Sons (1966); Michael O'Keeffe and Alexandra Navrotsky, eds., Structure and Bonding in Crystals II, Academic Press (1981), Chapters 19-22; and D. Pandey and P. Krishna, in Current Topics in Materials Science, vol. 9, E. Kaldis, ed., NorthHolland (1982), Chapter 2.

${ }^{15}$ C. Cheng, R.J. Needs, V. Heine, and N. Churcher, Europhys. Lett. 3, 475 (1987); C. Cheng, R.J. Needs, and Volker Heine, J. Phys. C 21, 1049 (1988); V. Heine, in Competing Interactions and Microstructures: Statics and Dynamics, R. LeSar, A. Bishop, and R. Heffner. eds. (Springer-Verlag, Berlin, 1987).

${ }^{16}$ A. Blandin, J. Friedel, and G. Saada, J. Phys. (Paris) Colloq. 27, C3-128 (1966); Charles W. Krause and J.W. Morris, Jr., Acta Met. 22, 767 (1974). 